\title{
Custos catastróficos em pacientes com tuberculose no Brasil: estudo em cinco capitais
}

\author{
Catastrophic costs in tuberculosis patients in Brazil: a study in five capitals \\ Costos catastróficos en pacientes con tuberculosis en Brasil: un estudio en cinco capitales
}

\author{
Leticia Molino Guidoni ${ }^{1}$ (D) \\ Leticya dos Santos Almeida Negri ${ }^{1}$ (1) \\ Geisa Fregona Carlesso ${ }^{1}$ (i) \\ Eliana Zandonade ${ }^{1}$ (a) \\ Ethel Leonor Noia Maciel ${ }^{1}$
}

1. Universidade Federal do Espírito Santo, Programa de Pós-Graduação em Saúde Coletiva, Laboratório de Epidemiologia. Vitória, ES, Brasil.
Autor correspondente:

Leticia Molino Guidoni.

E-mail: leticiaguidoni@hotmail.com

Recebido em 26/01/2021.

Aprovado em 02/06/2021.

DOl:https://doi.org/10.1590/2177-9465-EAN-2020-0546

\section{Resumo}

Objetivo: avaliar o impacto econômico domiciliar do adoecimento pela tuberculose no Brasil. Método: pesquisa transversa multicêntrica de 2016 a 2018, em cinco capitais do Brasil, em pacientes diagnosticados com tuberculose. Os custos totais foram a soma dos custos diretos e indiretos incorridos antes e durante o diagnóstico e tratamento. A regressão logística foi utilizada para estudar determinantes de custos catastróficos. A pobreza foi medida como renda familiar per capita diária < U\$ 5,5. Resultados: trezentos e sessenta e um pacientes foram inscritos no estudo. O custo extrapolação foi de $R \$ 3.664,47$ (DP: $\mathrm{R} \$ 2.667,67$ ) e o custo total de extrapolação foi de $\mathrm{R} \$ 22.291,82$ (DP: $\mathrm{R} \$ 16.259,50)$. No geral, $29 \%$ dos participantes do estudo foram caracterizados como pobres antes da tuberculose, e $39 \%$ depois. Em média, a renda diminuiu em $11 \%$ dos participantes e $41 \%$ tiveram custos catastróficos. Os determinantes estatisticamente significativos de experimentar custos catastróficos foram: participante ser o chefe da família, vivendo na pobreza antes da tuberculose, desemprego e interrupção do trabalho durante o tratamento $(p<0,05)$. Conclusão e implicações para a prática: embora o tratamento seja financiado pelo governo, a tuberculose continua resultando em custos catastróficos e diminuição da renda para muitas famílias no Brasil.

Palavras-chave: Tuberculose; Terapêutica; Custos e Análise de Custo; Estudos Transversais; Brasil.

\section{Abstract}

Objective: to assess the home economics impact of illness from Tuberculosis in Brazil. Method: multicenter cross-sectiona research from 2016 to 2018, in five capitals of Brazil, in patients diagnosed with tuberculosis. The total costs were the sum of the direct and indirect costs incurred before and during diagnosis and treatment. Logistic regression was used to study determinants of catastrophic costs. Poverty was measured as daily household income per capita < U $\$ 5.5$. Results: 361 patients were enrolled in the study. The extrapolation cost was $\mathrm{R} \$ 3,664.47$ (SD: $\mathrm{R} \$ 2,667.67$ ) and the total extrapolation cost was $\mathrm{R} \$ 22,291.82$ (SD: $\mathrm{R} \$ 16,259.50)$. Overall, $29 \%$ of study participants were characterized as poor before tuberculosis, and $39 \%$ afterwards. On average, income declined by $11 \%$ among participants and $41 \%$ had catastrophic costs. The statistically significant determinants of experiencing catastrophic costs were: participant being the head of the family; living in poverty before tuberculosis; unemployment and interruption of work during treatment $(p<0.05)$. Conclusion and implications for practice: although treatment is financed by the government, tuberculosis continues to cause catastrophic costs and decreased of income for many families in Brazil.

Keywords: Tuberculosis; Therapeutics; Costs and Cost Analysis; Cross-Sectional Studies; Brazil.

\section{Resumen}

Objetivo: evaluar el impacto económico domiciliario de enfermarse por tuberculosis en Brasil. Método: Investigación multicéntrica transversal de 2016 a 2018, en cinco capitales de Brasil, en pacientes diagnosticados con tuberculosis. Los costos totales fueron la suma de los costos directos e indirectos incurridos antes y durante el diagnóstico y el tratamiento. Se utilizó la regresión logística para estudiar los determinantes de los costos catastróficos. La pobreza se midió como un ingreso familiar diario per cápita < U\$ 5,5. Resultados: se inscribieron 361 pacientes en el estudio. El costo de extrapolación fue de R\$ 3.664,47 (DE: $R \$ 2.667,67)$ y el costo total de extrapolación fue $R \$ 22.291,82$ (DE: $R \$ 16.259,50)$. En general, el $29 \%$ de los participantes se caracterizaron como pobres antes de la tuberculosis y el 39\%, después. En promedio, los ingresos disminuyeron en $11 \%$ de los participantes y el $41 \%$ tuvo costos catastróficos. Determinantes estadísticos de costos catastróficos: participante ser cabeza de familia; vivir en pobreza antes de la tuberculosis; desempleo; interrupción del trabajo durante el tratamiento $(p<0.05)$. Conclusión e implicaciones para la práctica: aunque el tratamiento es financiado por el gobierno, la tuberculosis sigue teniendo costos catastróficos y una disminución de los ingresos para muchas familias en Brasil.

Palabras clave: Tuberculosis; Terapéutica; Costos y Análisis de Costo; Estudios Transversales; Brasil. 


\section{INTRODUÇÃO}

O Brasil, para evitar que os domicílios afetados pela Tuberculose (TB) experimentem dificuldades financeiras, trabalha com duas estratégias importantes para a saúde e renda da população ${ }^{1-2}$. No entanto, a TB continua associada à pobreza no Brasil e no mundo ${ }^{3-8}$. Em 2016, estima-se que 1,6 milhão de mortes por TB e 11,4 milhões de novos casos de TB ocorreram em países em desenvolvimento ${ }^{9}$. Os gastos com prevenção, diagnóstico e tratamento atingiram U\$ 6,6 bilhões globais em 2015, contra U\$ 6,2 bilhões, em 2014 ${ }^{10}$. As estimativas atualizadas, em 2019, para financiamento de prevenção, diagnóstico e tratamento no total para 2018-2022 são de U\$ 60 bilhões (uma média de U\$ 12 bilhões por ano) para os países em desenvolvimento ${ }^{11}$.

Apesar do tratamento gratuito, as pessoas com TB experimentam altos custos que, às vezes, podem levar à diminuição da renda ${ }^{5-6,12}$. Os custos totais enfrentados pelas pessoas com TB são compostos por despesas diretas com medicamentos, internação, transporte e alimentação, bem como custos indiretos da perda de renda relacionada à incapacidade de trabalhar durante a doença, devido ao tempo gasto no cuidado, estigma e discriminação. Tanto os custos diretos quanto indiretos são incorridos antes e após o diagnóstico de TB, podendo ser incorridos por pacientes, bem como outros familiares que prestam assistência e apoio ${ }^{13}$. Além dos custos diretos ou indiretos, as famílias afetadas pela TB também podem sofrer custos de enfrentamento do uso de poupanças preventivas e empréstimos de amigos e parentes ${ }^{13}$

Após o lançamento da Comissão dos Determinantes Sociais da Saúde, em 2008, a Organização Mundial da Saúde (OMS) intensificou seu foco nos determinantes sociais da saúde ${ }^{14}$. Isso reflete em sua estratégia de "End TB" que endossa a proteção social, o alívio da pobreza e ações sobre outros determinantes da TB. Um dos indicadores para o progresso na estratégia "EndTB"é a redução do número de famílias afetadas pelos custos catastróficos totais devidos à TB até $2020^{15}$. A OMS define os custos catastróficos totais devidos à TB como custos totais incorridos pelas famílias relacionadas à TB superiores a $20 \%$ da renda familiar anual ${ }^{13}$.

Estudos representativos nacionais que caracterizam os custos domésticos relacionados à TB têm sido realizados em vários países, incluindo Vietnã, Uganda, Tailândia, Gana e Indonésia ${ }^{3-6,12,16}$. No Brasil, estudos já pesquisaram os custos dos pacientes com TB, no entanto foram focados apenas em uma cidade ou um estado ${ }^{17-19}$. Nesse sentido, o estudo teve o objetivo de avaliar a ocorrência de custo catastrófico devido à TB em cinco capitais, uma em cada região do Brasil.

\section{MÉTODO}

Realizou-se um estudo transversal multicêntrico entre junho de 2016 e junho de 2018 em 14 unidades de saúde localizadas em cinco capitais, uma em cada região do Brasil (Manaus, Recife, Vitória, Campo Grande e Porto Alegre).

O cenário do estudo foi o Brasil, que está entre os 20 países com alta carga de TB e, em 2019, notificou 73.864 casos de TB incidente. ${ }^{20}$ Fatores como densidade populacional, raça e índice de pobreza são determinantes para altas taxas de TB no País. ${ }^{21}$ No Brasil, o tratamento da TB é integrado ao sistema universal de saúde financiado pelo governo que fornece todos os exames diagnósticos, antimicrobianos e, quando necessário, serviços hospitalares gratuitamente ${ }^{20}$.

Os dados foram obtidos através do questionário adaptado transculturalmente do manual de custo do paciente proposto pela $\mathrm{OMS}^{13,22-23}$. O questionário final coletou dados sobre as informações socioeconômicas dos participantes (por exemplo, renda), despesas de tratamento antes do diagnóstico de TB, no diagnóstico e durante o tratamento da TB, bem como o tempo perdido na busca ou acesso ao cuidado com a TB ${ }^{22-23}$. As entrevistas foram realizadas individualmente, nas próprias unidades selecionadas.

Foram critérios de inclusão para composição da amostra: indivíduos diagnosticados com TB com idade igual ou superior a 18 anos, há pelo menos 14 dias com tratamento sensível para o Esquema Básico (rifampicina, isoniazida, pirazinamida e etambutol por 2 meses e rifampicina e isoniazida por mais 4 meses) e que relataram renda familiar antes e depois da TB.

Para o cálculo da amostra, utilizamos a taxa de incidência da doença considerada no ano de 2012, segundo dados do Sistema de Informação de Agravos de Notificação atualizado em dezembro de 2013. Foi necessário um tamanho amostral de 330 para estimar a proporção de custos e análise de custos catastróficos entre a população estudada, com precisão de $5 \%$, assumindo perda de $20 \%$, proporção de $40 \%$ de custos catastróficos e efeito de design de 1,7.

Os participantes da pesquisa foram mostrados para as cinco capitais selecionadas proporcionalmente à notificação de TB em cada capital do estado. Prevendo possíveis perdas durante a obtenção dos dados de custos aumentou-se a amostra em $10 \%$, obtendo um tamanho amostral de 363 participantes, sendo realizada a coleta de dados com 361 participantes. Dos 361 pacientes elegíveis, 11 foram excluídos por não terem relatado a renda familiar antes e depois da TB, constituindo uma amostra de 350 pacientes.

Para análise de custo direto relacionado à TB, utilizamos os custos pré-diagnóstico e do tratamento. Os custos diretos pré-diagnóstico incluíram despesas autorreferidas em consultas, testes e medicamentos subtraindo qualquer reembolso de seguro, bem como viagens, alimentação e acomodação para acesso aos serviços de saúde. Os custos diretos durante o tratamento incluíram despesas de acesso ao Tratamento Diretamente Observada (TDO); retirada de medicamentos anti-TB; testes de acompanhamento; internação; e a compra de alimentos especiais.

$\mathrm{O}$ custo indireto para o paciente devido à incapacidade de trabalho foi a estimativa da renda mensal autorreferida antes da TB, multiplicada pelo número de meses que os participantes relataram parar o trabalho devido adoecimento.

Análise de custos totais relacionados à TB foi realizada conforme recomendado pela OMS e os custos foram coletados para um período de um mês. Essa abordagem requer cálculos para estimar os custos totais. Em primeiro lugar, os custos autorreferidos dos participantes foram extrapolados para o restante da fase atual de tratamento (fase intensiva: 2 meses; 
fase de manutenção: 4/6 meses). Em segundo lugar, os custos para a outra fase do tratamento foram imputados utilizando-se o custo médio relatado dos participantes da pesquisa entrevistados nessa fase. Para mais detalhes, consulte "WHO's Tuberculosis Patient Cost Surveys: A Handbook"13.

Os custos de enfrentamento foram a soma do dinheiro emprestado multiplicado pela taxa de juros reportada, mais a diferença de preço e valor dos imóveis vendidos.

Para a análise das dificuldades financeiras relacionadas à TB, medimos custos e dificuldades financeiras de duas maneiras, custos catastróficos e diminuição da renda. Para o cálculo dos custos catastróficos devido à TB, utilizamos a soma do total de custos diretos e indiretos dividido pelo valor da renda familiar anual antes do adoecimento por TB. Classificamos os participantes com custos totais $\geq 20 \%$ da renda familiar anual como presença de custo catastrófico. Para o cálculo da diminuição da renda por TB, subtraímos a renda familiar mensal autorreferida antes e depois da doença. Para caracterização da pobreza, classificamos as famílias como pobres e não pobres antes e depois da TB, utilizando o limite de renda familiar do Banco Mundial de U\$ 5,5 dólares por dia, recomendado para países desenvolvidos e subdesenvolvidos, como o Brasil24.

Análise estatística foi realizada utilizando-se o STATA 14.0. Resumimos os custos dos participantes, as características socioeconômicas e o número de participantes passando por dificuldades financeiras. $O$ teste qui-quadrado $(x 2)$ foi utilizado para comparar dados discretos, e a análise do teste de variância (ANOVA) foi utilizada para comparar dados contínuos. O coeficiente kappa de concordância e teste mcNemar foi usado para testar a consistência na notificação do estado de pobreza antes e depois da TB. A regressão logística foi utilizada para investigar fatores de risco para a experiência de custos catastróficos. Fatores de risco hipotéticos associados aos desfechos em valor $p<0,2$ foram incluídos na análise multivariável. Testamos a sensibilidade dos custos catastróficos ao utilizar três outros limites de custos $\geq$ $10 \%, \geq 15 \%$ e $\geq 25 \%$ da renda familiar anual.

O projeto foi aprovado pelo comitê de ética em pesquisa do Centro de Ciências da Saúde da Universidade Federal do Espírito Santo (CEP/UFES) (1.856.319/2016), e comitês de cada uma das capitais em estudo. Todos os participantes assinaram um Termo de Consentimento livre e Esclarecido.

\section{RESULTADOS}

Características descritivas. Dos 350 participantes do estudo $226(65 \%)$ era do sexo masculino, e em trabalho informal $211(60 \%)$. A maioria não tinha plano de saúde privado (277 / 79\%) (Tabela 1).

Custos relacionados à TB. O custo médio de extrapolação foi de $R \$ 3.664,47$ (DP: R\$2.667,67) O custo médio total de extrapolação foi de R $\$ 22.291,82$ (DP: R \$ 16.259,50) (Tabela 2).

Dificuldades financeiras relacionadas à TB. Aproximadamente $41 \%$ da amostra experimentou custos catastróficos devido à TB $\geq 20 \%$. Quarenta participantes (11\%) passaram de não pobre antes da TB para pobre depois da TB. A estatística kappa e o teste de McNemar foram, estatisticamente, significativos ( $p<0,001)$ (Tabela 3).

Determinantes de custos catastróficos devido à TB. Na regressão logística multivariável, os quatro determinantes seguintes estiveram significativamente associados à experiência de custos catastróficos: paciente chefe da família (aOR: 2,01 (1,23-3,28); $P=0,005)$, vivendo em situação de pobreza antes da TB (aOR: 2,65 (1,22-5,76); $P=0,014)$, emprego informal atual (aOR: 1,48 (1,13-1,94); $P=0,004)$, e parada do trabalho durante a doença (aOR: 1,72 (1,03-2,84); $\mathrm{P}=0,035)$ (Tabela 4).

Análise de sensibilidade. Foram utilizados limiares de custo catastrófico de $10 \%, 15 \%, 20 \%$ e $25 \%$ e dos participantes que apresentaram custo catastrófico nos respectivos limiares foram considerados pobres 85 (84\%), 71 (70\%), 61 (60\%) e 44 (44\%), respectivamente (Tabela 5 ).

\section{DISCUSSÃO}

Verificou-se que 145 (41\%) dos pacientes com TB experimentaram custos catastróficos e houve um aumento de $11 \%$ na probabilidade de viver em situação de pobreza durante o tratamento em relação à pré-doença. $O$ principal contribuinte para os custos totais foram os custos indiretos. Quatro determinantes foram associados à experiência de custos catastróficos: paciente chefe da família, viver na pobreza antes da TB, emprego informal atual e parar o trabalho durante a doença.

Os resultados desse estudo sugerem que o impacto econômico da TB nos pacientes e seus familiares, no Brasil, continua sendo grave $^{25}$. Esse achado é consistente com estudos anteriores no Brasil e no mundo. Em 2005, constatou-se que famílias em Salvador, Brasil, comprometeram cerca de $33 \%$ de sua renda familiar para custear o diagnóstico e o tratamento daTB. A perda de renda por incapacidade para o trabalho foi o fator que mais contribuiu para o ônus econômico das famílias com TB ${ }^{18}$. Em 2010, outro estudo, no Brasil, mostrou que os custos incorridos pelos pacientes com TB eram elevados, especialmente para aqueles em TDO e observou um aumento nos custos durante a fase de tratamento para aqueles em TDO, enquanto o tratamento autoadministrado foi associado a custos relativamente mais elevados durante a fase de pré-diagnóstico ${ }^{17}$. No presente estudo, os indicadores da situação financeira dos pacientes foram os principais determinantes para custos catastróficos. Esses achados são consistentes com estudos anteriores e destacam a necessidade de proteção financeira adicional para famílias afetadas por $\mathrm{TB}^{16}$.

O ponto forte do estudo foi que ele amostrava pacientes de cada uma das cinco regiões do Brasil. A representatividade da amostraé confirmada pela semelhança das características sociodemográficas sumárias com o registro nacional de TB eletrônica Sistema de Informação de Agravos de Notificação de 2014 a $2016^{26}$.

Embora a cobertura universal de saúde esteja disponível em todo o Brasil, este estudo indica que os pacientes com TB ainda estão em risco de dificuldades financeiras. Isso indica que há necessidade de intervenções governamentais para custear os custos relacionados à TB. No Peru, uma intervenção socioeconômica específica da TB voltada para famílias pobres 
Tabela 1. Características dos participantes do estudo das cinco Regiões do Brasil no período de junho/2016 a julho/2018 $(n=350)$.

\begin{tabular}{|c|c|c|c|c|}
\hline \multirow{2}{*}{ Variáveis } & \multirow{2}{*}{ Total } & \multicolumn{2}{|c|}{ Custo catastrófico } & \multirow{2}{*}{$p$-valor } \\
\hline & & Não (\%) & $\operatorname{Sim}(\%)$ & \\
\hline \multicolumn{5}{|l|}{ Gênero } \\
\hline Masculino & $226(65)$ & $137(60)$ & 89 (39) & \\
\hline Feminino & $124(35)$ & $68(55)$ & $56(45)$ & 0,294 \\
\hline \multicolumn{5}{|l|}{ Escolaridade } \\
\hline Analfabeto & $19(5)$ & $10(53)$ & $9(47)$ & 0,260 \\
\hline Ensino Fundamental & $154(44)$ & $83(54)$ & $71(46)$ & \\
\hline Ensino médio & $138(40)$ & $85(62)$ & $53(38)$ & \\
\hline Ensino Superior & 39 (11) & $27(69)$ & $12(31)$ & \\
\hline \multicolumn{5}{|l|}{ Idade (anos) } \\
\hline $18-40$ & $165(47)$ & $93(56)$ & $72(44)$ & 0,275 \\
\hline$>40$ & $182(52)$ & $109(60)$ & $73(40)$ & \\
\hline Não informado & $3(1)$ & $3(100)$ & 0 & \\
\hline \multicolumn{5}{|l|}{ Raça } \\
\hline Branco & $91(26)$ & $56(62)$ & $35(38)$ & 0,622 \\
\hline Preto & $55(16)$ & $31(56)$ & $24(44)$ & \\
\hline Amarelo & $7(2)$ & $3(43)$ & $4(57)$ & \\
\hline Pardo & $178(50)$ & $107(60)$ & $71(40)$ & \\
\hline Indígena & $17(5)$ & $7(41)$ & $10(59)$ & \\
\hline Não informado & $2(1)$ & $1(50)$ & $1(50)$ & \\
\hline \multicolumn{5}{|l|}{ Chefe da família } \\
\hline Paciente & $162(46)$ & $86(53)$ & $76(47)$ & 0,053 \\
\hline Não paciente & $188(54)$ & $119(63)$ & $69(37)$ & \\
\hline \multicolumn{5}{|l|}{ Trabalho formal } \\
\hline Sim & $139(40)$ & $97(70)$ & $42(30)$ & 0,001 \\
\hline Não & $211(60)$ & $108(51)$ & $103(49)$ & \\
\hline \multicolumn{5}{|c|}{ Parar de trabalhar/estudar/ devido à tuberculose } \\
\hline Sim & $218(63)$ & $116(53)$ & $102(47)$ & 0,017 \\
\hline Não & $126(36)$ & $86(68)$ & $40(32)$ & \\
\hline Não informado & $6(2)$ & $3(50)$ & $3(50)$ & \\
\hline \multicolumn{5}{|l|}{ Plano de Saúde } \\
\hline Sim & $73(21)$ & $49(67)$ & $24(33)$ & 0,095 \\
\hline Não & $277(79)$ & $156(56)$ & $121(44)$ & \\
\hline \multicolumn{5}{|l|}{ Tipo de TB } \\
\hline Pulmonar & $263(75)$ & $161(61)$ & $102(39)$ & 0,081 \\
\hline Extrapulmonar & $87(25)$ & $44(51)$ & $43(49)$ & \\
\hline \multicolumn{5}{|l|}{ Fase do tratamento } \\
\hline Intensiva & $98(28)$ & $51(52)$ & $47(48)$ & 0,122 \\
\hline Manutenção & $252(72)$ & $154(61)$ & 98 (39) & \\
\hline
\end{tabular}

Fonte: Banco de dados do estudo.

Legenda: TB - tuberculose / HIV - vírus da imunodeficiência humana. 
Tabela 1. Continuação...

\begin{tabular}{|c|c|c|c|c|}
\hline \multirow{2}{*}{ Variáveis } & \multirow{2}{*}{ Total } & \multicolumn{2}{|c|}{ Custo catastrófico } & \multirow{2}{*}{$p$-valor } \\
\hline & & Não (\%) & $\operatorname{Sim}(\%)$ & \\
\hline \multicolumn{5}{|l|}{ HIV } \\
\hline Positivo & $60(17)$ & $33(55)$ & $27(45)$ & 0,377 \\
\hline Negativo & $241(69)$ & $139(58)$ & $102(42)$ & \\
\hline Não testado/desconhecido & 49 (14) & $33(67)$ & $16(33)$ & \\
\hline \multicolumn{5}{|l|}{ Hospitalização } \\
\hline $\operatorname{sim}$ & $140(40)$ & $77(55)$ & $63(45)$ & 0,268 \\
\hline Não & $210(60)$ & $128(61)$ & $82(39)$ & \\
\hline \multicolumn{5}{|l|}{ Tratamento anterior para TB } \\
\hline Sim & $60(17)$ & $29(48)$ & $31(52)$ & 0,077 \\
\hline Não & $290(83)$ & $176(61)$ & $114(39)$ & \\
\hline \multicolumn{5}{|l|}{ Primeiro atendimento } \\
\hline Hospital público & $67(19)$ & $42(63)$ & $25(37)$ & 0,817 \\
\hline Hospital ou clínica privada & $22(7)$ & $14(64)$ & $8(36)$ & \\
\hline Unidade básica de saúde & $247(70)$ & $141(57)$ & $106(43)$ & \\
\hline Outros & $14(4)$ & $8(57)$ & $6(43)$ & \\
\hline \multicolumn{5}{|l|}{ Comorbidades } \\
\hline Sim & $142(41)$ & $81(57)$ & $61(43)$ & 0,631 \\
\hline Não & $208(59)$ & $124(60)$ & $84(40)$ & \\
\hline TOTAL & $350(100)$ & 205 & 145 & \\
\hline
\end{tabular}

Fonte: Banco de dados do estudo.

Legenda: TB - tuberculose / HIV - vírus da imunodeficiência humana.

Tabela 2. Distribuição dos custos relacionados à Tuberculose dos participantes das cinco Regiões do Brasil, no período de junho/2016 a julho/2018 $(n=350)$.

\begin{tabular}{|c|c|c|c|c|}
\hline Custos & Média (R\$) & DP & Mín & Máx \\
\hline Custos diagnóstico e pré-diagnóstico & 209,65 & 598,99 & $(0-$ & $6.800,01)$ \\
\hline Custos TDO & 5,85 & 34,62 & $(0-$ & $460,98)$ \\
\hline Custos com retirada de medicação & 40,39 & 151,10 & $(0-$ & $1.650,00)$ \\
\hline Custos de consultas & 8,37 & 47,04 & $(0-$ & $600,00)$ \\
\hline Custos de Hospitalização & 38,68 & 314,65 & $(0-$ & $4.499,98)$ \\
\hline Custos com alimentação & 106,51 & 229,81 & $10-$ & $2.999,99)$ \\
\hline Reembolso do tratamento & 6,51 & 65,14 & $10-$ & $899,99)$ \\
\hline Custos diretos do paciente & 193,24 & 533,93 & $(0-$ & $7.503,51)$ \\
\hline Custos indiretos & $3.442,22$ & $4.220,16$ & $(0-$ & $23.134,37)$ \\
\hline Custos diretos e indiretos & $3.634,79$ & $4.304,72$ & $10-$ & $23.138,43)$ \\
\hline Custos extrapolamento por mês & $3.664,47$ & $2.667,67$ & $(534,14-$ & $16.599,97)$ \\
\hline Total de custos de extrapolamento & $22.291,82$ & $16.259,50$ & $(3.204,85$ - & $99.599,95)$ \\
\hline Custos de enfrentamento & 339,54 & $2.508,84$ & (0- & $35.000,00)$ \\
\hline Renda familiar mensal & $2.574,29$ & $3.196,94$ & (0 - & $27.000,02)$ \\
\hline Renda familiar anual pré-doença & $30.891,67$ & $38.363,12$ & $(0-$ & $32.000,01)$ \\
\hline
\end{tabular}

Fonte: Banco de dados do estudo

Legenda: DP - desvio padrão / Mín - mínimo / Máx - máximo / TDO - tratamento diretamente observado. 


\section{Custos catastróficos na tuberculose}

Guidoni LM, Negri LSA, Carlesso GF, Zandonade E, Maciel ELN

Tabela 3. Resumo do estado de pobreza doméstica antes e depois da Tuberculose dos participantes das cinco Regiões do Brasil no período de junho/2016 a julho/2018 $(n=350)$.

\begin{tabular}{cccc}
\hline \multirow{2}{*}{ Variáveis } & \multirow{2}{*}{ Total } & \multicolumn{2}{c}{ Renda familiar após TB } \\
\cline { 3 - 4 } & & Pobre & Não pobre \\
\hline Pobre & $101(29)$ & $98(71)$ & $3(1)$ \\
\hline Não pobre & $249(71)$ & $40(29)$ & $209(99)$ \\
Total & 350 & $138(39)$ & $212(61)$ \\
\hline
\end{tabular}

Fonte: Banco de dados do estudo

Kappa $=0,7302(p<0,001)$ McNemar $p<0,001$

Legenda: TB - tuberculose.

Tabela 4. Determinantes de custos catastróficos relacionados à Tuberculose no Brasil dos participantes das cinco Regiões do Brasil no período de junho/2016 a julho/2018 ( $n=350)$.

\begin{tabular}{|c|c|c|c|c|c|c|c|c|c|c|c|}
\hline \multirow{2}{*}{ Nível } & \multirow{2}{*}{ Grupo } & \multirow{2}{*}{ Determinante } & \multirow{2}{*}{ Categoria } & \multicolumn{8}{|c|}{ Modelo multivariável } \\
\hline & & & & OR & $\mathrm{LI}$ & LS & $p$-valor & $\mathrm{aOR}$ & $\mathrm{LI}$ & LS & $p$-valor \\
\hline \multirow{4}{*}{ Distal } & \multirow{4}{*}{ sociodemográfico } & \multirow{2}{*}{ Escolaridade (anos) } & $0-8$ & 0,67 & 0,43 & 1,05 & 0,070 & 0,95 & 0,84 & 1,08 & 0,465 \\
\hline & & & $>8$ & 1,00 & & & & 1,00 & & & \\
\hline & & \multirow{2}{*}{ Chefe da família } & Paciente & 1,52 & 0,97 & 2,39 & 0,053 & 2,01 & 1,23 & 3,28 & 0,005 \\
\hline & & & Não paciente & 1,00 & & & & 1,00 & & & \\
\hline \multirow{6}{*}{ Intermediário } & \multirow{6}{*}{ Doença } & \multirow{2}{*}{ Fase do tratamento } & Intensiva & 1,44 & 0,87 & 2,38 & 0,121 & 1,51 & 0,89 & 2,55 & 0,120 \\
\hline & & & Manutenção & 1,00 & & & & 1,00 & & & \\
\hline & & \multirow{2}{*}{ Tipo de TB } & Pulmonar & 0,65 & 0,38 & 1,08 & 0,081 & 0,53 & 0,30 & 0,92 & 0,026 \\
\hline & & & Extrapulmonar & 1,00 & & & & 1,00 & & & \\
\hline & & \multirow{2}{*}{ Tratamento anterior para TB } & Sim & 1,65 & 0,90 & 3,00 & 0,077 & 1,77 & 0,95 & 3,29 & 0,068 \\
\hline & & & Não & 1,00 & & & & 1,00 & & & \\
\hline \multirow{10}{*}{ Proximal } & \multirow{10}{*}{ Financeiro } & \multirow{2}{*}{ Renda antes da TB } & Pobre & 2,99 & 1,80 & 4,87 & 0,000 & 2,65 & 1,22 & 5,76 & 0,014 \\
\hline & & & Não pobre & 1,00 & & & & 1,00 & & & \\
\hline & & \multirow{2}{*}{ Renda depois da TB } & Pobre & 2,29 & 1,44 & 3,65 & 0,000 & 1,06 & 0,50 & 2,26 & 0,871 \\
\hline & & & Não pobre & 1,00 & & & & 1,00 & & & \\
\hline & & \multirow{2}{*}{ Trabalho formal } & Sim & 1,00 & & & & 1,00 & & & \\
\hline & & & Não & 2,20 & 1,37 & 3,55 & 0,000 & 1,48 & 1,13 & 1,94 & 0,004 \\
\hline & & \multirow{2}{*}{ Parou de trabalhar } & Sim & 1,89 & 1,16 & 3,08 & 0,006 & 1,72 & 1,03 & 2,84 & 0,035 \\
\hline & & & Não & 1,00 & & & & 1,00 & & & \\
\hline & & \multirow{2}{*}{ Plano de Saúde } & Sim & 1,00 & & & & 1,00 & & & \\
\hline & & & Não & 0,63 & 0,35 & 1,11 & 0,095 & 1,04 & 0,53 & 2,00 & 0,907 \\
\hline
\end{tabular}

Fonte: Banco de dados do estudo

Legenda: LI - limite inferior / LS - limite superior/ OR - odds ratio / aOR - odds ratio ajustado / TB - tuberculose.

Tabela 5. Prevalência de custos catastróficos em diferentes limiares. Os resultados são apresentados, no geral, e para os participantes que vivem em agregados domiciliares pobres versus não pobres, das cinco Regiões do Brasil no período de junho/2016 a julho/2018 $(n=350)$.

\begin{tabular}{|c|c|c|c|c|}
\hline Custos totais catastróficos & \multirow{2}{*}{$10 \%$} & \multirow{2}{*}{$15 \%$} & \multirow{2}{*}{$20 \%$} & \multirow{2}{*}{$25 \%$} \\
\hline Agregados domiciliares & & & & \\
\hline Geral & $235(67 \%)$ & $180(51 \%)$ & $145(41 \%)$ & $103(29 \%)$ \\
\hline Pobre & 85 (84\%) & $71(70 \%)$ & $61(60 \%)$ & $44(44 \%)$ \\
\hline Não pobre & $150(60 \%)$ & $109(44 \%)$ & $84(32 \%)$ & $59(24 \%)$ \\
\hline $\mathrm{p}$-valor & 0,000 & 0,000 & 0,000 & 0,000 \\
\hline
\end{tabular}

Fonte: Banco de dados do estudo 
foi acessível a essa população e reduziu sua probabilidade de experimentar custos catastróficos ${ }^{27}$. No Brasil, a adoção da proteção social governamental devido a TB resistente aos medicamentos está associada à redução da probabilidade de enfrentar dificuldades financeiras. ${ }^{28}$

Um estudo de modelagem econômica em sete países de baixa e média renda indica que as transferências de dinheiro, provavelmente, reduzirão o fardo dos custos da TB para as famílias afetadas ${ }^{29}$.

Os custos que contribuíram para que 145 famílias enfrentem custos catastróficos durante o diagnóstico e tratamento da TB foram os custos diretos com transporte, alimentação e especialmente perda de renda. Esse risco é maior em pacientes de famílias pobres, pois têm maior risco de interrupção do trabalho e do estudo e isso pode estar relacionado a doenças mais graves por atraso no tratamento e deve ser melhor investigado. $O$ agregado familiar não pobre e com emprego formal pode ter acesso ao auxíliodoença ${ }^{30}$ e, consequentemente, parar de trabalhar e receber o subsídio de doença durante os cuidados. As famílias mais pobres podem não ter acesso ao auxílio-doença, porque podem não ter carteira assinada, sendo assim não podem parar de trabalhar. Não parar o trabalho é potencialmente ruim, pois pode afetar a probabilidade do paciente de concluir o tratamento com sucesso.

Embora os dados mostrem que os pobres têm apresentaram mais custo catastrófico (60\%), os não pobres também sofreram custo catastrófico (32\%), isso pode ser explicado pelo fato de que eles poderiam gastar algum dinheiro para agilizar o diagnóstico e ter uma situação mais confortável enquanto buscam o diagnóstico e terapêutica, além de condições para enfrentar a necessidade de alimentos adicionais enquanto se recuperam da doença (se eles têm dinheiro eles podem gastá-lo em busca de melhores condições de tratamento). Esses achados sugerem que os regimes de proteção social, como cesta de alimentos e vale-transporte, são um regime de proteção social que deve ser realizado para pacientes de famílias pobres. No Brasil, várias medidas de proteção social já estão em vigor. ${ }^{2}$

Serviços de saúde gratuitos são necessários, mas não suficientes no Brasil, a forma como o programa de TB é organizado pode precisar de uma melhoria para melhorar o atendimento rápido e eficaz aos pacientes com TB. Além disso, encontrar e tratar as pessoas mais pobres é o maior desafio, pois o foco na população vulnerável exige diferentes abordagens que normalmente não estão sob a competência padrão dos profissionais de saúde no Brasil.

$O$ auxílio financeiro aos pacientes que perderam a renda devido à TB não está totalmente sob a responsabilidade do Ministério da Saúde, e reduzir os custos para essas famílias afetadas pela TB, no Brasil, exigirá colaboração entre o Ministério da Saúde e o Ministério do Desenvolvimento Social. A criação de um sistema de referência de pacientes para o Sistema Único de Assistência Social realizado pelo Sistema Único de Saúde (SUS) será o próximo passo para o combate à TB no Brasil. Essa combinação é uma nova abordagem que precisa ser mais inovadora e focar nessa população vulnerável com cada vez mais dificuldades financeiras devido à TB, como um caminho importante para a eliminação da TB até 2035.

\section{CONCLUSÕES E IMPLICAÇÕES PARA A PRÁTICA}

Para nosso conhecimento, esta é a primeira pesquisa no Brasil a estimar a proporção de custos catastróficos entre as pessoas com TB sensível a todos os medicamentos. Os resultados fornecem uma linha de base para monitorar o progresso em direção à meta da OMS de garantir que nenhuma família afetada por TB enfrente dificuldades financeiras devido à doença e ajudam a identificar quais famílias precisam de apoio.

Os domicílios, no Brasil, correm o risco de passar por dificuldades financeiras devido ao adoecimento pela TB, apesar do acesso à Atenção Básica disponível pelo SUS. Os custos catastróficos e a diminuição da renda para muitas famílias, no Brasil, observados durante o tratamento da TB podem dificultar o acesso aos serviços de saúde e, consequentemente, o tratamento desses doentes.

Com relação as implicações para a prática, os achados da presente investigação têm potencial para direcionar estratégias de intervenção específicas para famílias de baixa renda que necessitam de um olhar diferenciado devido a situação financeira que já se encontram. Recomenda-se que a enfermagem atue assertivamente no acompanhamento dessas famílias, buscando manter o tratamento durante o tempo necessário para a promoção da cura.

O estudo também teve algumas limitações. Em primeiro lugar, os custos relacionados à TB foram autorreferidos, e os participantes podem ter experimentado a dificuldade em lembrar os custos incorridos antes do diagnóstico. Os participantes que trabalham no setor informal, autônomo e agrícola também podem ter dificuldade em estimar rendas. Em segundo lugar, não conseguimos avaliar os custos para aqueles pacientes que não podiam se dar ao luxo de procurar tratamento, como os sem-teto que poderia subestimar nossa proporção de custos catastróficos. Finalmente, o desenho transversal do estudo significa que podemos ter subestimado o uso de estratégias de enfrentamento, já que o risco dessa atividade provavelmente se acumulará durante o tratamento.

Importante destacar a necessidade do desenvolvimento de novas pesquisas com participantes que não possuem acesso ao sistema de saúde, bem como um desenho de estudo de acompanhamento desses gastos mensalmente, uma vez que o desenho transversal do estudo, pode ter subestimado o uso de estratégias de enfrentamento e custos, pois o risco tende a se acumular ao longo do tratamento.

\section{CONTRIBUIÇÕES DOS AUTORES}

Desenho do estudo. Leticia Molino Guidoni. Leticya dos Santos Almeida Negri. Geisa Fregona Carlesso. Eliana Zandonade. Ethel Leonor Noia Maciel.

Aquisição, análise de dados e interpretação dos resultados. Leticia Molino Guidoni. Leticya dos Santos Almeida Negri. Geisa Fregona Carlesso. Eliana Zandonade. Ethel Leonor Noia Maciel.

Redação e revisão crítica do manuscrito. Leticia Molino Guidoni. Leticya dos Santos Almeida Negri. Geisa Fregona Carlesso. Eliana Zandonade. Ethel Leonor Noia Maciel. 
Aprovação da versão final do artigo. Leticia Molino Guidoni. Leticya dos Santos Almeida Negri. Geisa Fregona Carlesso. Eliana Zandonade. Ethel Leonor Noia Maciel.

Responsabilidade por todos os aspectos do conteúdo e a integridade do artigo publicado. Leticia Molino Guidoni. Leticya dos Santos Almeida Negri. Geisa Fregona Carlesso. Eliana Zandonade. Ethel Leonor Noia Maciel.

\section{EDITOR ASSOCIADO}

Gerson Marinho (1)

\section{EDITOR CIENTÍFICO}

Marcelle Miranda da Silva (1)

\section{REFERÊNCIAS}

1. Sistema Único de Saúde (SUS): estrutura, princípios e como funciona (BR). Ministério da Saúde [site na internet], Brasília (DF), 04 jan 2013 [citado 08 jun 2020]. Disponivel em: https://www.saude.gov.br/sistemaunico-de-saude

2. Sistema Único de Assistência Social (SUAS): o que é (BR). Ministério da Cidadania, Secretaria Especial do Desenvolvimento Social [site na internet], Brasília (DF), 07 jan 2020 [citado 08 jun 2020]. Disponível em: http://mds.gov.br/assuntos/assistencia-social/o-que-e

3. Nhung NV, Hoa NB, Anh NT, Anh LTN, Siroka A, Lönnroth Ket al. Measuring catastrophic costs due to tuberculosis in Vietnam. Int J Tuberc Lung Dis. 2018 set;22(9):983-90. http://dx.doi.org/10.5588/ijtld.17.0859. PMid:30092862.

4. Pedrazzoli D, Siroka A, Boccia D, Bonsu F, Nartey K, Houben R et al. How affordable is TB care? Findings from a nationwide TB patient cost survey in Ghana. Trop Med Int Health. 2018 ago;23(8):870-8. http:// dx.doi.org/10.1111/tmi.13085. PMid:29851223.

5. Muttamba W, Tumwebaze R, Mugenyi L, Batte C, Sekibira R, Nkolo A et al. Households experiencing catastrophic costs due to tuberculosis in Uganda: magnitude and cost drivers. BMC Public Health. 2020;20(1):1409. http://dx.doi.org/10.1186/s12889-020-09524-5. PMid:32938411.

6. Fuady A, Houweling TAJ, Mansyur M, Burhan E, Richardus JH. Effect of financial support on reducing the incidence of catastrophic costs among tuberculosis-affected households in Indonesia: eight simulated scenarios. Infect Dis Poverty. 2019;8(1):10. http://dx.doi.org/10.1186/ s40249-019-0519-7. PMid:30709415.

7. Tomeny EM, Mendoza VL, Marcelo DB, Barrameda AJD, Langley I, Abong JM et al. Patient-cost survey for tuberculosis in the context of patient-pathway modelling. Int J Tuberc Lung Dis. 2020 abr;24(4):420-7. http://dx.doi.org/10.5588/ijtld.19.0206. PMid:32317067.

8. Oxlade O, Murray M. Tuberculosis and poverty: why are the poor at greater risk in India? PLoS One. 2012;7(11):e47533. http://dx.doi. org/10.1371/journal.pone.0047533. PMid:23185241.

9. World Health Organization. WHO Report 2016. Global tuberculosis control. Geneva:WHO; 2017 [citado 08 jun 2020]. Disponível em: https:// apps.who.int/iris/handle/10665/250441

10. World Health Organization. WHO Report 2015. Global tuberculosis control. Geneva:WHO; 2015 [citado 08 jun 2020]. Disponível em: https:// apps.who.int/iris/bitstream/handle/10665/191102/9789241565059_ eng.pdf?sequence $=1$

11. World Health Organization. WHO Report 2019. Global tuberculosis control. Geneva: WHO; 2019 [citado 08 jun 2020]. Disponível em: https://apps. who.int/iris/bitstream/handle/10665/329368/978924156571 4-eng.pdf

12. Tanimura T, Jaramillo E, Weill D, Raviglione M, Lonnroth K. Financial burden for tuberculosis patients in low-and middle-income countries: a systematic review. Eur Respir J. 2014;43(6):1763-75. http://dx.doi. org/10.1183/09031936.00193413. PMid:24525439.

13. World Health Organization. Tuberculosis patient cost surveys: a handbook. Geneva:WHO; 2017 [citado 08 jun 2020]. Disponível em: https://apps. who.int/iris/bitstream/handle/10665/259701/9789241 513524-eng pdf? sequence $=1$

14. Nhlema BM, Kemp J, Steenbergen G, Theobald S, Tang S, Squire SB Systematic analysis of TB and poverty. Geneva: WHO; 2003.

15. World Health Organization. The end TB strategy. Geneva: WHO; 2015 [citado 08 jun 2020]. Disponível em: https://www.who.int/tb/strategy/ End_TB_Strategy.pdf?ua=1

16. Fuady A, Houweling TAJ, Mansyur M, Richardus JH. Catastrophic total costs in tuberculosis-affected households and their determinants since Indonesia's implementation of universal health coverage. Infect Dis Poverty. 2018 jan;7(1):3. http://dx.doi.org/10.1186/s40249-017-0382-3. PMid:29325589.

17. Steffen R, Menzies D, Oxlade O, Pinto M, de Castro AZ, Monteiro P et al Patients' costs and cost-effectiveness of tuberculosis treatment in DOTS and non-DOTS facilities in Rio de Janeiro, Brazil. PLoS One.2010;5(11):e14014. http://dx.doi.org/10.1371/journal.pone.0014014. PMid:21103344.

18. Costa JG, Santos AC, Rodrigues LC, Barreto ML, Roberts JA Tuberculosis in Salvador, Brazil: costs to health system and families. Rev Saude Publica. 2005;39(1):122-8. http://dx.doi.org/10.1590/S003489102005000100016 . PMid: 15654469.

19. Prado TN, Wada N, Guidoni LM, Golub JE, Dietze R, Maciel EL. Costeffectiveness of community health worker versus home-based guardians for directly observed treatment of tuberculosis in Vitória, Espírito Santo State, Brazil. Cad Saude Publica. 2011;27(5):944-52. http://dx.doi. org/10.1590/S0102-311X2011000500012. PMid:21655845.

20. Ministério da Saúde (BR). Manual de recomendações para o controle da tuberculose no Brasil. Brasília (DF): Ministério da Saúde; 2019 [citado 08 jun 2020]. Disponível em: https://bvsms.saude.gov.br/bvs/publicacoes/ manual_recomendacoes_controle_tuberculose_brasil_2_ed.pdf

21. Harling G, Castro MC. A spatial analysis of social and economic determinants of tuberculosis in Brazil. Health Place. 2014;25:56-67. http://dx.doi.org/10.1016/j.healthplace.2013.10.008. PMid:24269879.

22. KNCV Tuberculosis Foundation. The tool to estimate patients' costs Maanweg: KNCV; 2008 [citado 08 jun 2020]. Disponível em: http:// www.stoptb.org/wg/dots_expansion/tbandpoverty/assets/documents/ Tool\%20to\%20estimate\%20Patients'\%20Costs.pdf

23. Nunes GF, Guidoni LM, Negri LSA et al. Adaptação transcultural do instrumento "Tool to estimate patient's costs" em municípios prioritários do Brasil no controle da tuberculose. Esc Anna Nery. 2018;22(4):e20180073.

24. Instituto Brasileiro de Geografia e Estatística. Síntese de indicadores sociais: uma análise das condições de vida da população brasileira. Rio de Janeiro: IBGE; 2017 [citado 08 jun 2020]. Disponível em: http:// renastonline.ensp.fiocruz.br/sites/default/files/arquivos/recursos/ liv101459.pdf.

25. Wingfield T, Boccia D, Tovar M, Gavino A, Zevallos K, Montoya R et al. Defining catastrophic costs and comparing their importance for adverse tuberculosis outcome with multi-drug resistance: a prospective cohort study, Peru. PLoS Med. 2014 jul;11(7):e1001675. http://dx.doi. org/10.1371/journal.pmed.1001675. PMid:25025331.

26. Dados tuberculose no Brasil (BR). Sistema de Informação de Agravos de Notificação [site na internet], Brasília (DF), 2018 [citado 08 jun 2020]. Disponível em: http://portalsinan.saude.gov.br/tuberculose

27. Wingfield T, Tovar MA, Huff D, Boccia D, Montoya R, Ramos E et al. The economic effects of supporting tuberculosis-affected households in Peru. Eur Respir J. 2016 nov;48(5):1396-410. http://dx.doi. org/10.1183/13993003.00066-2016. PMid:27660507.

28. Rudgard WE, Chagas NS, Gayoso R, Barreto ML, Boccia D, Smeeth L et al. Uptake of governmental social protection and financial hardship during drug-resistant tuberculosis treatment in Rio de Janeiro, Brazil. Eur Respir J. 2018 mar;51(3):1800274. http://dx.doi.org/10.1183/13993003.002742018. PMid:29567727.

29. Rudgard WE, Evans CA, Sweeney S, Wingfield T, Lönnroth K, Barreira $D$ et al. Comparison of two cash transfer strategies to prevent catastrophic costs for poor tuberculosis-affected households in low- and middle-income countries: an economic modelling study. PLoS Med. 2017;14(11):e1002418. http://dx.doi.org/10.1371/journal.pmed.1002418. PMid:29112693.

30. E-gestor: Atenção Básica. Informação e gestão da Atenção Básica. Ministério da Saúde [plataforma na internet], Brasília (DF), 2020 [citado 08 jun 2020]. Disponível em: https://egestorab.saude.gov.br/ 